\title{
NOUVELle
}

\section{Rôle du trafic membranaire dans la migration cellulaire Une nouvelle application pour les neurotoxines clostridiales?}

Véronique Proux-Gillardeaux, Thierry Galli
Trafic membranaire et Morphogenèse neuronale et épithéliale, Équipe Avenir Inserm, Institut Jacques Monod, 2, place Jussieu, 75005 Paris, France. thierry@tgalli.net la cellubrévine également appelée VAMP3, dans la migration cellulaire $[3,4]$. Les protéines SNARE sont localisées au niveau des vésicules de transport (v-SNARE, v pour vésiculaire) ou au niveau de la membrane de chaque
> La migration cellulaire est un mécanisme fondamental au cours de l'embryogenèse, mais aussi à l'âge adulte, notamment pour le recrutement de cellules spécialisées telles que les macrophages aux sites d'infection ou pour la réparation des tissus lésés. Pour migrer, les cellules acquièrent un phénotype polarisé que l'on compare souvent à une forme en « escargot ». Le noyau et la majorité du cytoplasme se trouvent vers l'arrière de la cellule alors qu'à l'avant, une longue extension beaucoup plus fine formant un large lamellipode correspond au front de migration. Lors de la migration cellulaire, la cellule étend son front de migration, trouve une direction à suivre par chimiotactisme et le front de migration adhère à nouveau avant que les points d'ancrage devenus inutiles ne se détachent. La cellule se rétracte alors de l'arrière vers l'avant.

Deux modèles ont été proposés pour expliquer le mouvement du front de migration cellulaire : le premier accorde un rôle prépondérant au cytosquelette et on sait notamment que la polymérisation de l'actine est nécessaire pour pousser le front de migration. Le second modèle propose que l'exocytose et l'endocytose jouent un rôle fondamental dans ce processus $[1,2]$. Une exocytose régulée pourrait apporter des membranes et participer à l'extension des bords cellulaires. Elle pourrait aussi apporter les récepteurs nécessaires au chimiotactisme et à l'attachement des cellules aux substrats. En parallèle, l'endocytose pourrait réguler localement les réponses des récep- teurs membranaires, notamment ceux impliqués dans le chimiotactisme et ceux nécessaires à l'adhérence des cellules au substrat, ou permettre le recyclage des récepteurs de l'arrière de la cellule vers le front de migration cellulaire.

\section{Rôle des protéines SNARE}

Dans les cellules eucaryotes, le trafic membranaire permet l'acheminement des protéines et des lipides entre les différents compartiments cellulaires délimités par des membranes lipidiques. II met en jeu les protéines SNARE (soluble N-ethyl maleimide [NEM]-sensitive fusion protein [NSF] attachment protein receptor). Deux études récentes mettent en évidence le rôle d'une protéine SNARE endosomique, organite cible ( $\mathrm{t}$-SNARE, $\mathrm{t}$ pour target). La formation de complexes $v$-/t-SNARE particuliers permet la fusion de la membrane des vésicules avec la membrane de leur compartiment accepteur. Les neurotoxines tétanique et botuliques produites par les bactéries clostridiales sont responsables du tétanos et $\mathrm{du}$ botulisme [5]. Les chaînes légères de ces toxines sont de puissants inhibiteurs de la libération de neurotransmetteurs car elles protéolysent certaines protéines SNARE neuronales, la synaptobrévine (v-SNARE) ou ses t-SNARE partenaires, syntaxine 1 et SNAP-25 [6]. Les cellules non neuronales expriment la cellubrévine, homologue de la synaptobrévine, aussi sensible à la toxine tétanique.

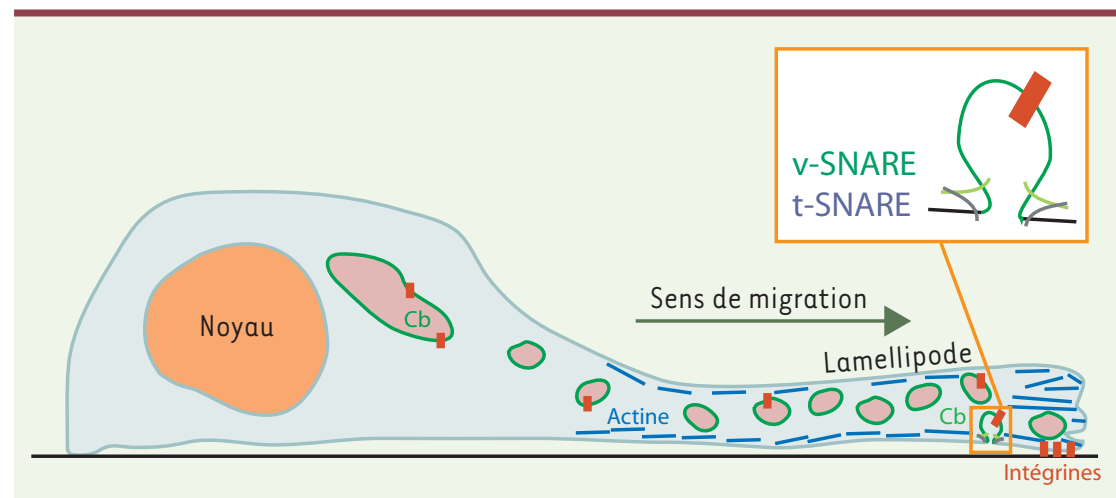

Figure 1. Rôle de la cellubrévine dans la migration d'une cellule épithéliale. Les vésicules endosomiques portant la cellubrévine sont responsables du recyclage des intégrines au niveau du front de migration de la cellule, régulant ainsi l'adhérence et la migration. La toxine tétanique inactive la cellubrévine en la protéolysant. L'actine joue aussi un rôle important dans ces phénomènes. L'encart montre comment la cellubrévine, une protéine v-SNARE, est impliquée dans la fusion des vésicules de sécrétion avec la membrane plasmique, en formant un complexe SNARE avec un t-SNARE. 


\section{Cellubrévine et toxine tétanique}

Le rôle de la cellubrévine ( $\mathrm{Cb}$ ) dans la migration cellulaire a été mis en évidence grâce à la toxine tétanique en exprimant sa chaîne légère dans des cellules épithéliales. La migration cellulaire est étudiée en observant la fermeture de blessures réalisées mécaniquement sur des monocouches de ces cellules. Des calculs de vitesse de migration réalisés lors de la fermeture de blessures montrent que les cellules exprimant la toxine tétanique (TeNT) sauvage ont une vitesse de migration réduite de moitié par rapport aux cellules exprimant une forme mutée de la toxine tétanique. La cellubrévine joue donc un rôle important dans la migration cellulaire [3]. L'équipe de M.G. Coppolino (Guelph, ON, Canada) est arrivée à cette même conclusion dans des cellules fibroblastiques et en faisant des tests de migration par chimiotactisme [4]. Au cours de la migration, comme nous l'avons vu précédemment, les cellules doivent réguler en permanence leur adhérence au substrat. La migration nécessite une dynamique complexe et finement régulée d'interactions entre la cellule et la matrice extracellulaire, que ce soit au niveau du front de migration ou à l'arrière de la cellule.
La Cb colocalise partiellement avec des marqueurs de points focaux comme la FAK (focal adhesion kinase) et la Taline. De plus, les cellules exprimant la TeNT sauvage adhèrent à des substrats de type collagène mettant en jeu la $\beta 1$-intégrine plus rapidement que celles exprimant une TeNT inactive. Les intégrines sont des hétérodimères formés par des sousunités transmembranaires de type $\alpha$ et $\beta$ médiant les interactions avec la matrice extracellulaire. Les cellules en migration, en bordure de blessure, exprimant la TeNT mutante, contrairement à celles exprimant la TeNT sauvage, internalisent un anticorps anti- $\beta 1$-intégrine. Dans ces cellules, la $\mathrm{Cb}$ colocalise avec la $\beta 1$-intégrine endocytée [3]. La Cb joue donc un rôle important dans la migration cellulaire en régulant l'adhérence des cellules au substrat médiée par la $\beta 1$-intégrine.

En résumé, l'expression de la toxine tétanique protéolysant spécifiquement la cellubrévine ralentit de moitié la vitesse de migration des cellules. La cellubrévine régule l'adhérence des cellules au substrat en participant à l'exocytose et à l'endocytose des intégrines. Le rôle important de la cellubrévine dans la migration cellulaire ouvre de nouvelles perspectives dans le cadre de la cancérothérapie, suggérant que le trafic membranaire pourrait être une nouvelle cible pour inhiber la migration des cellules métastatiques. Les neurotoxines clostridiales, déjà utilisées en cosmétologie (réduction de rides) et pour soigner des spasmes musculaires en neurologie et en ophtalmologie, pourraient ainsi trouver de nouvelles applications en cancérothérapie. $\nabla$

Tetanus neurotoxin-mediated cleavage of cellubrevin impairs epithelial cell migration and integrin-dependent cell adhesion

\section{RÉFÉRENCES}

1. Bretscher MS. Moving membrane up to the front of migrating cells. Cell $1996 ; 85: 465-7$.

2. Bretscher MS, Aguado-Velasco C. Membrane traffic during cell locomotion. Curr Opin Cell Biol 1998; 10 : 537-41.

3. Proux-Gillardeaux V, Gavard J, Irinopoulou T, et al. Tetanus neurotoxin-mediated cleavage of cellubrevin impairs epithelial cell migration and integrindependent cell adhesion. Proc Natl Acad Sci USA 2005; $102: 6362-7$.

4. Tayeb MA, Skalski M, Cha MC, et al. Inhibition of SNARE-mediated membrane traffic impairs cell migration. Exp Cell Res 2005 ; 305 : 63-73.

5. Montecucco C, Schiavo G. Mechanism of action of tetanus and botulinum neurotoxins. Mol Microbiol $1994 ; 13: 1-8$.

6. Galli T, Martinez Arca S, Paumet F. Mécanisme de la fusion membarnaire. Med Sci (Paris) 2002 ; 18 1113-9.

\section{NOUVELle}

\section{La galectine-7 : un nouveau gène associé au pouvoir métastatique}

Mélanie Demers, Yves St-Pierre
INRS (Institut national de la recherche scientifique)Institut Armand-Frappier (IAF), Université du Québec, 531, boulevard des Prairies, Laval, Québec, H7V IB7 Canada.

yves.st-pierre@

> Les lymphomes constituent un groupe hétérogène de pathologies caractérisées par la transformation néoplasique de cellules lymphoïdes. Ils se divisent en deux catégories : le lymphome hodgkinien classique (ou maladie de Hodgkin) et les lymphomes non-hodgkiniens (LNH), un groupe hétérogène de lymphomes. Dans le premier cas, les tumeurs sont, la plupart du temps, peu nombreuses et très localisées et, de plus, croissent relativement lentement. Au contraire, la plupart des LNH sont très agressifs et se propagent aux organes lymphoïdes, tels que les ganglions et la rate, de même que vers les organes non lymphoïdes. Les protocoles thérapeutiques visant à contrer la croissance et la propagation de ces cellules reposent essentiellement sur une polychimiothérapie dont l'intensité et la particularité dépendent du type de la tumeur et de l'âge du patient. L'identification de nouveaux gènes associés au développement des formes agressives des cancers lymphoïdes représente donc une étape importante dans la lutte contre cette maladie. 